Pacific Journal of Mathematic 


\title{
CONTRACTOR DIRECTIONS, DIRECTIONAL CONTRACTORS AND DIRECTIONAL CONTRACTIONS FOR SOLVING EQUATIONS
}

\author{
Mieczyslaw Altman
}

\begin{abstract}
The concept of contractor directions appears to be a natural development of the concept of directional contractors. By using both concepts, sufficient conditions are presented for a nonlinear operator to be a mapping onto a Banach space.
\end{abstract}

Several authors have recently studied the solvability of nonlinear operator equations. Their main objective was to investigate a class of nonlinear operators having closed ranges. In the first part of this paper, a generalization of some of the known results is presented and, in addition, new proofs are given.

In the second part, a class of operators is discussed without assumption that the operators in question have closed ranges. In both parts, the technique of proofs is based on the concept of contractor directions. This concept is a generalization of a concept of asymptotic directions introduced by Browder. However, this generalization is rather closely related to the concept of directional contractors. By using the method of directional contractors, in the third part of this paper, a class of nonlinear operators with Hölder continuous Fréchet derivatives is investigated. Sufficient conditions are given which guarantee that the operators in question are mappings onto. Finally, as a generalization of contraction mappings, directional contractions are discussed with application to evolution equations.

Definition 1.1. Let $X$ be an abstract set and $P: X \rightarrow Y$ a mapping of $X$ into a (real or complex) Banach space $Y, x$ a point in $X$. Then we define sets $\Gamma_{x}(P)$ of contractor directions for $P$ at $x$, and $\Gamma_{x}(P)$ is such a set if there exists a positive $q<1$ with the following property: for each $y \in \Gamma_{x}(P)$, there exist a positive number $\epsilon=\epsilon(x, y) \leqq 1$ and an element $\bar{x} \in X$ such that

$$
\|P \bar{x}-P x-\epsilon y\| \leqq q \epsilon\|y\| \text {. }
$$

Thus, $\Gamma_{x}(P)=\Gamma_{x q}(P)$ depends on $q$ and obviously $\Gamma_{x q}(P) \subset \Gamma_{x \bar{q}}(P)$ if $0<q<\bar{q}<1$.

LEMMA 1.1. The set $\bar{\Gamma}_{x \bar{q}}(P)$ contains $\Gamma_{x q}(P)$, where $0<q<\bar{q}<1$. 
If $\Gamma_{x}(P)$ is dense in some sphere $S(0, r)=[y: y \in Y,\|y\| \leqq r]$, then $\Gamma_{x}(P)=Y$.

Proof. Let $0<q<\bar{q}<1$. If $v \in S(0, r)$ and $y \in \Gamma_{x q}(P)$ are such that $\|v-y\| \leqq \eta$, then

$$
\begin{aligned}
\|P \bar{x}-P x-\epsilon v\| & \leqq\|P \bar{x}-P x-\epsilon y\|+\epsilon y\|v-y\| \leqq q \epsilon\|y\|+\epsilon \eta \\
& \leqq q \epsilon(\|v\|+\eta)+\epsilon \eta \\
& \leqq \bar{q} \epsilon\|v\| \text { if } \eta \leqq\left(\bar{q}-q 1\|v\|(1+q)^{-1}\right. \\
& \text { in virtue of }(1.1) .
\end{aligned}
$$

Lemma 1.2. For all $x \in X$, let $y_{0}-P x$ be in the closure of $\Gamma_{x}(P)$, where $y_{0} \in Y$ is not in $P(X), P(X)$ being closed. Then there exists a sphere $S\left(y_{0}, r\right) \subset Y$ such that $y-P x \in \Gamma_{x}(P)$ for all $y \in S\left(y_{0}, r\right), x \in X$.

Proof. By Lemma 1.1, $y_{0}-P x \in \Gamma_{x}(P)$ for all $x \in X$. Thus, it follows that

$$
\left\|P \bar{x}-P x-\epsilon\left(y_{0}-P x\right)\right\| \leqq q \epsilon\left\|y_{0}-P x\right\|
$$

is satisfied for all $x \in X$ and some positive $q<1$. Hence, we obtain

$$
\begin{array}{r}
\|P \bar{x}-P x-\epsilon(y-P x)\| \leqq\left\|P \tilde{x}-P x-\epsilon\left(y_{0}-P x\right)\right\|+\epsilon\left\|y_{0}-y\right\| \\
\leqq q \epsilon\left\|y_{0}-P x\right\|+\epsilon r \leqq q \epsilon(\|y-P x\|+r)+\epsilon r \leqq \bar{q} \epsilon\|y-P x\|
\end{array}
$$

for all $y \in S\left(y_{0}, r\right), x \in X$, where $\bar{q}$ is arbitrary with $q<\bar{q}<1$ and $r$ is chosen so as to satisfy

$$
(q+1) r \leqq(\bar{q}-q) d \leqq(\bar{q}-q)\|y-P x\|,
$$

where $2 d$ is the distance from $y_{0}$ to the closed set $P(X)$.

The following two lemmas of Gavurin (see [3] for references) will be used in our considerations.

LEMMA 1.3. Let $\alpha$ be an ordinal number of first or second class and let $\left\{t_{\gamma}\right\}_{0 \leqq y \leqq \alpha}$ be a naturally well-ordered sequence of real numbers provided that for numbers $\beta$ of second kind we have

$$
t_{\beta}=\lim _{\gamma>\beta} t_{\gamma}
$$

Then the following equality holds. 


$$
t_{\alpha}=t_{0}+\sum_{0 \leqq \gamma<\alpha}\left(t_{\gamma+1}-t_{\gamma}\right)
$$

LEMMA 1.4. Let $\alpha$ be an ordinal number of first or second class and let $\left\{x_{\gamma}\right\}_{0 \leqq y \leqq \alpha}$ be a well-ordered sequence of elements of a metric space $X$ provided that for numbers $\beta$ of second kind we have

$$
x_{\beta}=\lim _{\gamma / \beta} x_{\gamma}
$$

Then, denoting by $d(\cdot, \cdot)$ the distance,

$$
d\left(x_{\alpha}, x_{0}\right) \leqq \sum_{0 \leqq \gamma<\alpha} d\left(x_{\gamma+1}, x_{\gamma}\right)
$$

THEOREM 1.1. Let $X$ be an abstract set, $Y$ a real or complex Banach space, $P: X \rightarrow Y$ a mapping of $X$ into $Y$ such that the range $P(X)$ is closed in $Y$. Suppose that $y_{0} \in Y$, which is not on the boundary of $P(X)$, is such that for each $x$ in $X$, the element $y_{0}-P x$ belongs to the closure of a set $\Gamma_{x}(P)$ of contractor directions for $P$ at $x$. Then there exists a solid sphere $S\left(y_{0}, r\right) \subset Y$ with center $y_{0}$ and radius $r$ such that the equation

$$
P x=y, y \in S\left(y_{0}, r\right), \quad x \in X
$$

has a solution for every $y \in S\left(y_{0}, r\right)$.

Proof. One can assume that $y_{0}$ is not in $P(X)$. Then, by Lemma 1.2, there exists a sphere $S\left(y_{0}, r\right)$ such that $y-P x \in \Gamma_{x}(P)$ for all $y \in S\left(y_{0}, r\right), x \in X$, i.e., there exist positive numbers $q<1, \epsilon=\epsilon(x, y) \leqq$ 1 and elements $\bar{x} \in X$ such that

$$
\|P \bar{x}-P x-\epsilon(y-P x)\| \leqq q \epsilon\|y-P x\|
$$

for all $y \in S\left(y_{0}, r\right), x \in X$. For the sake of simplicity, we replace equation (1.2) by $P x=0$ in which the same symbol $P$ stands for the new mapping with values $P x-y$, and fixed $y \in S\left(y_{0}, r\right)$. Then inequality (1.3) yields

$$
\|P \bar{x}-P x-\epsilon P x\| \leqq q \epsilon\|P x\|
$$

Now, we construct well-ordered sequences of numbers $t_{\alpha}$ and elements $P\left(t_{\alpha}\right) \in P(X)$ as follows.

Put $t_{0}=0$ and and let $x_{0}$ be an arbitrary element of $X$ and put $P\left(t_{0}\right)=P x_{0} \in P(X)$. Suppose that $t_{\gamma}$ and $P\left(t_{\gamma}\right)$ have been constructed 
for all $\gamma<\alpha$, provided that: for arbitrary number $\gamma<\alpha$ inequality $\left(1.5_{\gamma}\right)$ is satisfied.

$$
\left\|P\left(t_{\gamma}\right)\right\| \leqq e^{-(1-q) t_{\gamma}}\left\|P\left(t_{0}\right)\right\|, \quad P\left(t_{\gamma}\right) \in P(X)
$$

for first kind numbers $\beta=\gamma+1<\alpha$ the following inequalities are satisfied:

$\left(1.6_{\gamma+1}\right) \quad\left\|P\left(t_{\gamma+1}\right)-P\left(t_{\gamma}\right)\right\| \leqq(1+q)\left\|P\left(t_{0}\right)\right\| e^{-(1-q) t_{\gamma}}\left(t_{\gamma+1}-t_{\gamma}\right) ;$
$\left(1.7_{\gamma+1}\right)$
$0<t_{\gamma+1}-t_{\gamma} \leqq 1$

and for second kind numbers $\gamma<\alpha$ the following relations hold:

$$
t_{\gamma}=\lim _{\beta>\gamma} t_{\beta}, \quad P\left(t_{\gamma}\right)=\lim _{\beta>\gamma} P\left(t_{\beta}\right) .
$$

Then it follows from (1.6)-(1.8), Lemmas 1.3 and 1.4 that for arbitrary $\lambda<\gamma<\alpha$ we have

$$
\begin{aligned}
\left\|P\left(t_{\gamma}\right)-P\left(t_{\lambda}\right)\right\| & \leqq \sum_{\lambda \leqq \beta<\gamma}\left\|P\left(t_{\beta+1}\right)-P\left(t_{\beta}\right)\right\| \\
& \leqq(1+q)\left\|P\left(t_{0}\right)\right\| \sum_{\lambda \leqq \beta<\gamma} e^{-(1-q) t_{\beta}}\left(t_{\beta+1}-t_{\beta}\right) \\
& =(1+q)\left\|P\left(t_{0}\right)\right\| \sum_{\lambda \leqq \beta<\gamma} e^{(1-q)\left(t_{\beta+1}-t_{\beta}\right)} e^{-(1-q) t_{\beta+1}}\left(t_{\beta+1}-t_{\beta}\right) \\
& \leqq(1+q) e^{1-q} \sum_{\lambda \leqq \beta<\gamma} e^{-(1-q) t_{\beta+1}}\left(t_{\beta+1}-t_{\beta}\right) \\
& <(1+q) e^{1-q} \sum_{\lambda \leqq \beta<\gamma} \int_{t_{\beta}}^{t_{\beta+1}} e^{-(1-q) t} d t \\
& =(1+q) e^{1-q} \int_{t_{\lambda}}^{t_{\gamma}} e^{-(1-q) t} d t .
\end{aligned}
$$

Hence,

$$
\left\|P\left(t_{\gamma}\right)-P\left(t_{\lambda}\right)\right\| \leqq(1+q) e^{1-q} \int_{t_{\lambda}}^{t_{\gamma}} e^{-(1-q)} d t .
$$

Suppose that $\alpha$ is a first kind number. If $P\left(t_{\alpha-1}\right)=0$, then the proof of the theorem is completed, since $P\left(t_{\alpha-1}\right) \in P(X)$. Suppose now that $P\left(t_{\alpha-1}\right) \neq 0$, and let $x \in X$ be such that $P x=P\left(t_{\alpha-1}\right)$. Then there exist a positive $\epsilon \leqq 1$ and an element $\bar{x} \in X$ satisfying (1.4). Put $\tau_{\alpha}=\epsilon \leqq 1$ 


$$
t_{\alpha}=t_{\alpha-1}+\tau_{\alpha}, \quad P\left(t_{\alpha}\right)=P \bar{x} .
$$

Then we obtain by using (1.4) and the induction assumption $\left(1.5_{\alpha-1}\right)$

$$
\begin{aligned}
\left\|P\left(t_{\alpha}\right)\right\| & \leqq\left[1-(1-q) \tau_{\alpha}\right]\left\|P\left(t_{\alpha-1}\right)\right\|<e^{-(1-q) \tau_{\alpha}}\left\|P\left(t_{\alpha-1}\right)\right\| \\
& \leqq e^{-(1-q) \tau_{\alpha}} e^{-(1-q) t_{\alpha-1}}\left\|P\left(t_{0}\right)\right\|=e^{-(1-q) t_{\alpha}}\left\|P\left(t_{0}\right)\right\|,
\end{aligned}
$$

by (1.10). In virtue of (1.4), (1.10) we obtain

$$
\left\|P\left(t_{\alpha}\right)-P\left(t_{\alpha-1}\right)\right\| \leqq(1+q) \tau_{\alpha}\left\|P\left(t_{\alpha-1}\right)\right\| \leqq(1+q)\left\|P\left(t_{0}\right)\right\| e^{-(1-q) t_{\alpha-1}}\left(t_{\alpha}-t_{\alpha-1}\right) .
$$

Thus, conditions $\left(1.5_{\alpha}\right)-\left(1.7_{\alpha}\right)$ are satisfied for $t_{\alpha}$.

Now, suppose that $\alpha$ is a number of second kind and put $t_{\alpha}=$ $\lim _{\gamma>\alpha} t_{\gamma}$. Let $\left\{\gamma_{n}\right\}$ be an increasing sequence convergent to $\alpha$. It follows from (1.9) that $\left\{P\left(t_{\gamma_{n}}\right)\right\}$ is a Cauchy sequence and so is $\left\{P\left(t_{\gamma}\right)\right\}$. Denote by $P\left(t_{\alpha}\right)$ its limit. Since $P\left(t_{\gamma}\right)$ satisfy $\left(1.5_{\gamma}\right)$, it follows that $P\left(t_{\alpha}\right)$ satisfies $\left(1.5_{\alpha}\right)$. The process will terminate if $t_{\alpha}=+\infty$, where $\alpha$ is of second kind. In this case $\left(1.5_{\alpha}\right)$ yields $P\left(t_{\alpha}\right)=0$ and the proof is completed.

As a consequence of the above theorem we obtain

THEOREM 1.2. Let $X$ be an abstract set, $Y$ a real or complex Banach space, $P: X \rightarrow Y$ a mapping of $X$ into $Y$ such that the range $P(X)$ is closed in $Y$.

Suppose that for each $x$ in $X$, a set $\Gamma_{x}(P)$ of contractor directions is dense in $S_{r}=S(0, r) \subset Y$. Then $P(X)=Y$.

Proof. By Lemma 1.1, the set $\Gamma_{x}(P)$ contains the sphere $S_{r}$. Hence, it follows that $\Gamma_{x}(P)=Y$. Let $y_{0} \in Y$ be not in $P(X)$. Since $y_{0}-P x$ is in $\Gamma_{x}(P)$ for every $x \in X$, it follows from Theorem 1.1 that the equation $P x=y_{0}$ has a solution. This contradiction shows that $P(X)=Y$.

REMARK 1.1. Both theorems with some changes are proved in [4], for both real, vector space $X$ and Banach space $Y$. However, the proof given here is different and eliminates in [4] the use of a theorem by Zabreiko and Krasnosel'skii [9]. The above result presents a considerable generalization of a theorem by Browder [6] and of a theorem by Zabreiko and Krasnosel'skii [9], and yields, in addition, new proofs.

2. The restriction that the range of the operator in question is closed seems to play an essential role in the previous. discussion. However, by introducing the concept of special contractor directions the mentioned restriction can be removed. 
Definition 2.1. Let $X$ be a complete metric space, $Y$ be a real or complex Banach space, $P$ a mapping of $X$ into $Y, x$ a point in $X$. Then the element $y$ is said to belong to a set $\Gamma_{x}(P)$ of special contractor directions for $P$ at $x$ if there exist positive constants $B, q<1, \epsilon=$ $\epsilon(x, y) \leqq 1$ and an element $\bar{x} \in X$ such that

$$
\|P \bar{x}-P x-\epsilon y\| \leqq q \epsilon\|y\|,
$$

where $d(\bar{x}, x) \leqq B \epsilon\|y\|$. It is obvious that a special contractor direction is a contractor direction, and that Lemmas 1.1 and 1.2 are also valid here.

A mapping $P: D(P) \subset X \rightarrow Y$ is said to be closed if $x_{n} \rightarrow x$ and $P x_{n} \rightarrow y$ imply $x \in D(P)$ and $y=P x$.

THEOREM 2.1. Let $P: D(P) \subset X \rightarrow Y$ be a closed operator, $Y$ being a real or complex Banach space. Suppose that $y_{0} \in Y$ is such that for each $x$ in $D(P)$ the element $y_{0}-P x$ belongs to the closure of the set $\Gamma_{x}(P)$. Then the equation

$$
P x-y_{0}=0, \quad x \in D(P)
$$

has a solution. If, in addition, $y_{0}$ is not a limit point of $P(D(P))$, then there exists a solid sphere $S\left(y_{0}, r\right)$ such that the equation $P x=y$ has a solution for each $y \in S\left(y_{0}, r\right)$.

Proof. Without loss of generality, assume that $y_{0}=0$. We construct well-ordered sequences of numbers $t_{\alpha}$ and elements $x_{\alpha} \in D(P)$ as follows. Put $t_{0}=0$ and let $x_{0}$ be an arbitrary element of $D(P)$. Suppose that $t_{\gamma}$ and $x_{\gamma}$ have been constructed for all $\gamma<\alpha$, provided that: for arbitrary number $\gamma<\alpha$ inequality $\left(2.3_{\gamma}\right)$ is satisfied.

$$
\left\|P x_{\gamma}\right\| \leqq e^{-(1-q) t_{r}}\left\|P x_{0}\right\|
$$

for first kind numbers $\gamma+1<\alpha$ the following inequalities are satisfied:

$$
0<t_{\gamma+1}-t_{\gamma} \leqq 1
$$

$$
d\left(x_{\gamma+1}, x_{\gamma}\right) \leqq B\left\|P x_{0}\right\| e^{-(1-q) t_{\gamma}}\left(t_{\gamma+1}-t_{\gamma}\right),
$$

$$
\left\|P x_{\gamma+1}-P x_{\gamma}\right\| \leqq(1+q)\left\|P x_{0}\right\| e^{-(1-q) t_{\gamma}}\left(t_{\gamma+1}-t_{\gamma}\right)
$$

and for second kind numbers $\gamma<\alpha$ the following relations hold:

$$
t_{\gamma}=\lim _{\beta>\gamma} t_{\beta}, \quad x_{\gamma}=\lim _{\beta>\gamma} x_{\beta}, \quad P x_{\gamma}=\lim _{\beta>\gamma} P x_{\beta} .
$$


Then it follows from (2.5), (2.7), Lemmas 1.3 and 1.4 that for arbitrary $\gamma<\alpha$ and $\lambda<\gamma$ we have

$$
\begin{aligned}
& d\left(x_{\gamma}, x_{\lambda}\right) \leqq \sum_{\lambda \leqq \beta<\gamma} d\left(x_{\beta+1}, x_{\beta}\right) \leqq B\left\|P x_{0}\right\| \sum_{\lambda \leqq \beta<\gamma} e^{-(1-q) t_{\beta}}\left(t_{\beta+1}-t_{\beta}\right) \\
& =B\left\|P x_{0}\right\| \sum_{\lambda \leqq \beta<\gamma} e^{\left.(1-q) t_{\beta+1}-t_{\beta}\right)} e^{-(1-q) t_{\beta+1}}\left(t_{\beta+1}-t_{\beta}\right) \\
& <B\left\|P x_{0}\right\| e^{1-q} \sum_{\lambda \leqq \beta<\gamma} e^{-(1-q) t_{\beta+1}}\left(t_{\beta+1}-t_{\beta}\right) \\
& <B e^{1-q}\left\|P x_{0}\right\| \sum_{\lambda \leqq \beta<\gamma} \int_{t_{\beta}}^{t_{\beta+1}} e^{-(1-q) t} d t=B e^{1-q}\left\|P x_{0}\right\| \int_{t_{\lambda}}^{t_{\gamma}} e^{-(1-q) t} d t .
\end{aligned}
$$

Hence,

$$
d\left(x_{\gamma}, x_{\lambda}\right) \leqq B e^{1-q}\left\|P x_{0}\right\| \int_{t_{\lambda}}^{t_{\gamma}} e^{-(1-q) t} d t
$$

In the same way we obtain from (2.4), (2.6), (2.7), Lemmas 1.3 and 1.4 that

$$
\left\|P x_{\gamma}-P x_{\lambda}\right\| \leqq(1+q) e^{1-q}\left\|P x_{0}\right\| \int_{t_{\lambda}}^{t_{\gamma}} e^{-(1-q) t} d t
$$

Suppose that $\alpha$ is a first kind number. If $P x_{\alpha-1}=0$, then the proof of the first statement of the theorem is completed.

If $P x_{\alpha-1} \neq 0$, then we put

$$
t_{\alpha}=t_{\alpha-1}+\tau_{\alpha}, \quad x_{\alpha}=\bar{x},
$$

where $\bar{x} \in D(P)$ and $\tau_{\alpha}=\epsilon \leqq 1$ are chosen so as to satisfy (2.1) with $x=x_{\alpha-1}, y=y_{0}-P x_{\alpha-1}=-P x_{\alpha-1}$, since $y_{0}-P x_{\alpha-1}$ is a contractor direction for $P$, in virtue of Lemma 1.1. Thus, we have

$$
\left\|P x_{\alpha}-P x_{\alpha-1}+\tau_{\alpha} P x_{\alpha-1}\right\| \leqq q \tau_{\alpha}\left\|P x_{\alpha-1}\right\|
$$

Hence, we obtain, by (2.9) and $\left(2.3_{\alpha-1}\right)$,

$$
\begin{gathered}
\left\|P x_{\alpha}\right\| \leqq\left(1-\tau_{\alpha}\right)\left\|P x_{\alpha-1}\right\|+q \tau_{\alpha}\left\|P x_{\alpha-1}\right\|=\left(1-(1-q) \tau_{\alpha}\right)\left\|P x_{\alpha-1}\right\| \\
<e^{-(1-q) \tau_{\alpha}}\left\|P x_{\alpha-1}\right\| \leqq e^{-(1-q) t_{\alpha}}\left\|P x_{0}\right\|, \quad \text { i.e., } \\
\left\|P x_{\alpha}\right\| \leqq e^{-(1-q) t_{\alpha}}\left\|P x_{0}\right\| .
\end{gathered}
$$

It follows from $(2.10),(2.1)$ and $\left(2.3_{\alpha-1}\right)$ that 
$\left(2.13_{\alpha}\right) \quad d\left(x_{\alpha}, x_{\alpha-1}\right) \leqq B \tau_{\alpha}\left\|P x_{\alpha-1}\right\| \leqq B\left(t_{\alpha}-t_{\alpha-1}\right) e^{-(1-q) t_{\alpha-1}}\left\|P x_{0}\right\|$.

It follows from $(2.1),(2.11)$ and $\left(2.3_{\alpha-1}\right)$ that

$$
\begin{aligned}
\| P x_{\alpha} & -P x_{\alpha-1}\left\|\leqq(1+q) \tau_{\alpha}\right\| P x_{\alpha-1} \| \\
& \leqq(1+q)\left\|P x_{0}\right\| e^{-(1-q) t_{\alpha-1}}\left(t_{\alpha}-t_{\alpha-1}\right) .
\end{aligned}
$$

Thus, conditions $\left(2.3_{\alpha}\right),\left(2.4_{\alpha}\right),\left(2.5_{\alpha}\right)$, and $\left(2.6_{\alpha}\right)$ are satisfied for $t_{\alpha}$ and $x_{\alpha}$. Now suppose that $\alpha$ is a number of second kind and put $t_{\alpha}=$ $\lim _{\gamma>\alpha} t_{\gamma}$. Let $\left\{\gamma_{n}\right\}$ be an increasing sequence convergent to $\alpha$. It follows from (2.8) and (2.9) that $\left\{x_{\gamma_{n}}\right\}$ and $\left\{P x_{\gamma_{n}}\right\}$ are Cauchy sequences and so are $\left\{x_{\gamma}\right\}$ and $\left\{P x_{\gamma}\right\}$. Denote by $x_{\alpha}$ and $y_{\alpha}$ their limits, respectively. Since $P$ is closed we infer that $x_{\alpha} \in D(P)$ and $y_{\alpha}=P x_{\alpha}$. If $t_{\alpha}<+\infty$, then the limit passage in $\left(2.3_{\gamma_{n}}\right)$ yields $\left(2.3_{\alpha}\right)$. The relationships $\left(2.7_{\alpha}\right)$ are satisfied by the definition of $t_{\alpha}$ and $x_{\alpha}$, since $y_{\alpha}=P x_{\alpha}$. This process will terminate if $t_{\alpha}=+\infty$, where $\alpha$ is of second kind. In this case, $P x_{\alpha}=0$ in virtue of $\left(2.3_{\alpha}\right)$. Thus, the proof of the first assertion of the theorem is completed. To prove the remaining assertion we apply Lemma 1.2 which also holds true for special contractor directions. The proof then follows from the first assertion.

As a consequence of the above theorem we obtain

Theorem 2.2. Let $P: D(P) \subset X \rightarrow Y$ be a closed operator, where $X$ is a complete metric space and $Y$ is a real or complex Banach space. Suppose that for each $x \in D(P) \subset X$, the set $\Gamma_{x}(P)$ of special contractor directions for $P$ is dense in some sphere with center 0 in $Y$. Then $P(X)=Y$.

Proof. By Lemma 1.1 applied to special contractor directions, we have that $\Gamma_{x}(P)=Y$ for all $x \in X$. The proof follows from the first assertion of Theorem 2.1, since $y_{0}-P x \in \Gamma_{x}(P)$ for all $y_{0} \in Y$ and $x \in X$.

Using the method of contractor directions, a local existence theorem for nonlinear operator equations can also be proved.

Let $X_{0}$ be a subset of the complete metric space $X$. Put $S=$ $S\left(x_{0}, r\right)=\left[x: d\left(x, x_{0}\right)<r, x \in X\right]$ for a given $x_{0} \in X_{0}$, and $U=X_{0} \cap \bar{S}$, where $\bar{S}$ is the closure of $S$ in $X$. Let $P: U \rightarrow Y$ be a nonlinear operator closed on $U$, i.e., $x_{n} \in U, x_{n} \rightarrow x$ and $P x_{n} \rightarrow y$ imply that $x \in U$ and $y=P x$, where $y$ is an element of the Banach space $Y$.

THEOREM 2.3. Suppose that the following hypotheses are satisfied:

(1) $P: U \rightarrow Y$ is closed on $U$;

(2) for each $x \in U_{0}=X_{0} \cap S$, the set $\Gamma_{x}(P)$ of special contractor directions is dense in some sphere with center 0 in $Y$.

(3) $r \geqq B(1-\bar{q})^{-1}\left\|P x_{0}\right\|$, 
where $\bar{q}$ is arbitrary with $q<\bar{q}<1$, and $\bar{q}, B$ are defined by (2.1). Then equation $P x=0$ has a solution in $U$.

Proof. By Lemma 1.1, $\Gamma_{x}(P)=Y$ for all $x \in U_{0}$. Now, as in the proof of Theorem 2.1, we construct the sequences $\left\{t_{\alpha}\right\}$ and $\left\{x_{\alpha}\right\}$ satisfying the required induction assumptions and, in addition, instead of $\left(2.4_{\gamma+1}\right)$,

$$
0<\tau_{y+1}=t_{y+1}-t_{\gamma}<(1-q)^{-1} \ln (1-q)(1-\bar{q})^{-1},
$$

for first kind numbers $\beta=\gamma+1<\alpha$. Hence, we obtain the following estimate instead of $(2.8)$

$$
\begin{aligned}
d\left(x_{\gamma}, x_{\alpha}\right) & \leqq B\left\|P x_{0}\right\| \sum_{\lambda \leqq \beta<\gamma} e^{(1-q)\left(t_{\beta+1}-t_{\beta}\right)} e^{-(1-q) t_{\beta+1}}\left(t_{\beta+1}-t_{\beta}\right) \\
& <(1-q)(1-\bar{q})^{-1} B\left\|P x_{0}\right\| \sum_{\lambda \leqq \beta<\gamma} e^{-(1-q)\left(t_{\beta+1}-t_{\beta}\right)}\left(t_{\beta+1}-t_{\beta}\right) \\
& <(1-q)(1-\bar{q})^{-1} B\left\|P x_{0}\right\| \int_{t \lambda}^{t_{\gamma}} e^{-(1-q) t} d t,
\end{aligned}
$$

and in the same way we obtain instead of (2.9)

$$
\left\|P x_{\gamma}-P x_{\lambda}\right\| \leqq\left(1-q^{2}\right)(1-\bar{q})^{-1}\left\|P x_{0}\right\| \int_{t_{\lambda}}^{t_{\gamma}} e^{-(1-q) t} d t
$$

It follows, in particular, from the first estimate that

$$
d\left(x_{\gamma}, x_{0}\right)<(1-q)(1-\bar{q})^{-1} B\left\|P x_{0}\right\| \int_{0}^{\infty} e^{-(1-q) t} d t=(1-\bar{q})^{-1} B\left\|P x_{0}\right\| \leqq r .
$$

Hence, it follows that all elements $x_{\gamma}$ belong to $U_{0}$. The further reasoning is exactly the same as in the proof of Theorem 2.1.

Remark 2.1. Condition (3) in Theorem 2.3 can be replaced by

$$
r \geqq B(1-q)^{-1} e^{1-q}\left\|P x_{0}\right\| \text {. }
$$

In this case the induction assumption $\left(2.4_{\gamma+1}\right)$ remains without change, leading through (2.8) to the estimate

$$
d\left(x_{\gamma}, x_{0}\right)<B e^{1-q}\left\|P x_{0}\right\| \int_{0}^{\infty} e^{-(1-q) t} d t=B(1-q)^{-1} e^{1-q}\left\|P x_{0}\right\| \leqq r .
$$

The estimate (2.9) remains the same. 
The fact that the indicated replacement of $\left(2.4_{\gamma+1}\right)$ is feasible is based on the following remark.

REMARK 2.2. If the set $\Gamma_{x}(P)$ of special contractor directions is dense in some sphere with center 0 in $Y$, then for arbitrary $\tau>0, y \in Y$, there is a positive $\epsilon \leqq \tau$ satisfying condition (2.1).

In fact, since $\Gamma_{x}(P)=Y$, by Lemma 1.1 , for $\tau y$, there is an $\epsilon_{0}=$ $\epsilon_{0}(x, \tau y) \leqq 1$ satisfying (2.1) with $\epsilon=\epsilon_{0}$ and $y$ replaced by $\tau y$. Hence, (2.1) is satisfied with $\epsilon=\epsilon_{0} \tau \leqq \tau$.

Lemma 2.1. Let $A: X \rightarrow Y$ be a bounded linear operator, $X$ and $Y$ being Banach spaces. The following conditions are equivalent:

(i) there exist positive constants $B, q$ with $q<1$ such that for every $y \in Y$ there is an element $h \in X$ satisfying the inequalities

$$
\|A h-y\| \leqq q\|y\| \text { and }\|h\| \leqq B\|y\|
$$

(ii) A maps $X$ onto $Y$.

Proof. Put $x_{1}=h$ with $h$ satisfying (2.15) for $y=y_{0}$, and $x_{n+1}=$ $x_{n}-h_{n+1}$, where $h_{n+1}$ satisfies (2.15) for $y=A x_{n}-y_{0}$. Then we obtain, by $(2.15)$,

$$
\begin{aligned}
& \left\|x_{n+1}-x_{n}\right\|=\left\|h_{n+1}\right\| \leqq B\left\|A x_{n}-y_{0}\right\| \\
& =B\left\|A h_{n}-\left(A x_{n-1}-y_{0}\right)\right\| \leqq B q\left\|A x_{n-1}-y_{0}\right\|
\end{aligned}
$$$$
\leqq B q^{n}\left\|y_{0}\right\| \text {. }
$$

Hence, it follows that $\left\{x_{n}\right\}$ converges to some $x$ and $A x_{n} \rightarrow y_{0}=A x$, where $y_{0}$ is an arbitrary but fixed element of $Y$.

Lemma 2.2. Let $A: X \rightarrow Y$ be a bounded linear operator which maps the Banach space $X$ onto the Banach space $Y$, and let $B>\left\|\left(A^{*}\right)^{-1}\right\|$, where $A^{*}$ is the adjoint operator. Then for each $y \in Y$, there exists $h \in X$ such that $A h=y$ and $\|h\| \leqq B\|y\|$.

Proof. Denote by $\theta$ the set of all solutions of the equation $A x=0$. The transformation $A$ defines on the quotient space $X / \theta$ a bounded linear operator $\mathscr{A}: X / \theta \rightarrow Y$ by the formula $y=\mathscr{A} \xi$, where $x \in \xi \in X / \theta$ and $y=A x$. By Lemma [1], $\left\|\mathscr{A}^{-1}\right\|=\left\|\left(A^{*}\right)^{-1}\right\|$ (the domain of $\left(A^{*}\right)^{-1}$ is not, in general, the whole space). Since $\|\xi\| \leqq\left\|\mathscr{A}^{-1}\right\|\|y\|$, where $\|\xi\|=\inf [\|x\|: x \in \xi]$, there exists an element $x \in \xi$ such that $\|x\| \leqq\|\xi\|(1+\eta)$, where $\eta$ is determined by the relationship $\left\|\mathscr{A}^{-1}\right\|(1+\eta)=B$. Then we obtain 


$$
\|x\| \leqq\|\xi\|(1+\eta) \leqq\left\|\mathscr{A}^{-1}\right\|(1+\eta)\|y\|=B\|y\|,
$$

and $A x=y$.

As an application of Theorem 2.2 we obtain

Theorem 2.4. Let $P: X \rightarrow Y$ be a closed mapping of the Banach space $X$ into the Banach space $Y$. Suppose that $P$ is differentiable in the Gateaux sense and its derivative $P^{\prime}(x)$ maps $X$ onto $Y$ for each $x \in X$. If there exists a constant $B$ such that $\left\|\left(P^{\prime}(x)^{*}\right)^{-1}\right\| \leqq B$ for all $x \in X$, then $P(X)=Y$.

Proof. Let $y \in Y$ and $q$ be arbitrary with $0<q<1$. By Lemma 2.2 , there exists an element $h \in X$ such that

$$
P^{\prime}(x) h-y=0 \text { and }\|h\| \leqq \bar{B}\|y\|,
$$

where $\bar{B}>B$ is arbitrary. Choose a positive $\epsilon \leqq 1$ such that

$$
\left\|P(x+\epsilon h)-P x-\epsilon P^{\prime}(x) h\right\| \leqq q \epsilon\|y\| \text {. }
$$

Then we obtain

$$
\begin{aligned}
\|P(x+\epsilon h)-P x-\epsilon y\| \leqq & \left\|P(x+\epsilon h)-P x-\epsilon P^{\prime}(x) h\right\| \\
& +\epsilon\left\|P^{\prime}(x) h-y\right\| \leqq \epsilon q\|y\|
\end{aligned}
$$

and $\|\bar{x}-x\| \leqq \bar{B} \epsilon\|y\|$ is true for $\bar{x}=x+\epsilon h$, that is, condition (2.1) is satisfied. Hence, it follows that the set of special contractor directions $\Gamma_{x}(P)$ is the whole of $Y$ for all $x \in X$ and Theorem 2.2 is applicable.

A local existence theorem can be obtained on the basis of Theorem 2.3.

Let $X_{0}$ be a linear subset of the Banach space $X, S=S\left(x_{0}, r\right)$ for a given $x_{0} \in X_{0}$ and $U=X_{0} \cap \bar{S}$.

THEOREM 2.5. Let $P: U \rightarrow Y$ be closed on $U$. For each $x \in U_{0}=$ $X_{0} \cap S$, the Gâteaux derivative $P^{\prime}(x)$ exists and maps $X$ onto the Banach space $Y$. There exist positive constants $\bar{B}, \bar{q}<1$ such that $r>$ $\bar{B}(1-\bar{q})^{-1}\left\|P x_{0}\right\|$ and $\left\|\left(P^{\prime}(x)^{*}\right)^{-1}\right\| \leqq \bar{B}$ for all $x \in U_{0}$. Then equation $P x=0$ has a solution in $U$.

Proof. There exists $B>\bar{B}$ such that $r \geqq B(1-\bar{q})^{-1}\left\|P x_{0}\right\|$. By using the same argument as in the proof of Theorem 2.4, one can see that condition (2.1) is satisfied for arbitrary positive $q<\bar{q}$. Hence, it follows that the set $\Gamma_{x}(P)$ of special contractor directions is the whole of $Y$ for all $x \in U_{0}$. Thus, the hypotheses of Theorem 2.4 are satisfied. 
3. Definition [3]. Let $X$ be a vector space, $Y$ a Banach space and $P$ a mapping of $D(P) \subset X$ into $Y$. Then a linear mapping $\Gamma(x): Y \rightarrow X$ is said to be a directional contractor for $P$ at $x \in X$ if there exists a positive $q<1$ such that for every $y \in Y$, there is a positive number $\epsilon=\epsilon(x, y) \leqq 1$ satisfying the following inequality

$$
\|P(x+\epsilon \Gamma(x) y)-P x-\epsilon y\| \leqq q \epsilon\|y\| .
$$

It is assumed that $D(P)$ is linear and $\Gamma(x)(Y) \subset D(P)$ for all $x \in D(P)$. Denote by $L(Y \rightarrow X)$ be the set of all linear continuous mappings of $Y$ into $X$, where $X, Y$ are Banach spaces. Then $\Gamma: D(P) \rightarrow L(Y \rightarrow X)$ is called a contractor for $P$. In particular, if there exists a constant $B$ such that $\|\Gamma(x)\| \leqq B$ for all $x \in D(P)$, then $\Gamma$ is called a bounded irectional contractor for $P$.

THEOREM [3]. A closed nonlinear operator $P: D(P) \subset X \rightarrow Y$ which has a bounded directional contractor $\Gamma$ is a mapping onto $Y$.

Proof. This theorem is a particular case of Theorem 2.2. Let us notice that the condition imposed on $\epsilon$ in the contractor inequality (3.1) is less restrictive than that in [3].

The following lemma is obvious.

Lemma 3.1. Let $P: X \rightarrow Y$ be a nonlinear mapping of the Banach space $X$ into the Banach space $Y$. Suppose that $\Gamma(x): Y \rightarrow X$ is a bounded linear operator and that there exist positive numbers $r$ and $q<1$ such that

$$
\|P(x+\Gamma(x) y)-P x-y\| \leqq q\|y\|
$$

for all $y \in Y$ with $\|y\| \leqq r$. Then $\Gamma(x)$ is a directional contractor for $P$ at $x \in X$.

LEMMA 3.2. Let $P: X \rightarrow Y$ be a nonlinear operator differentiable in the Frèchet sense with Hölder continuous derivative $P^{\prime}(x)$, i.e., there exist positive numbers $K, \alpha \leqq 1$ such that

$$
\left\|P^{\prime}(x)-P^{\prime}(\bar{x})\right\| \leqq K\|x-\bar{x}\|^{\alpha}
$$

for all $x, \bar{x} \in X$. Moreover, for every $x \in X$, let $A(x): X \rightarrow Y$ be a bounded linear nonsingular operator such that

$$
\left\|A(x)^{-1}\right\| \leqq B \quad \text { and } \quad\left\|P^{\prime}(x)-A(x)\right\| \leqq C, B C<1
$$


for all $x \in X$ and some constants $B, C$. Then $\Gamma$ defined by $\Gamma(x)=A(x)^{-1}$ is a bounded directional contractor for $P$.

Proof. We have, by (3.3) and (3.4),

$$
\begin{gathered}
\|P(x+\Gamma(x) y)-P x-y\| \leqq\left\|P(x+\Gamma(x) y)-P x-P^{\prime}(x) \Gamma(x) y\right\| \\
+\left\|P^{\prime}(x) \Gamma(x) y-A(x) \Gamma(x) y\right\| \leqq(1+\alpha)^{-1} K\|\Gamma(x) y\|^{1+\alpha} \\
+C\|\Gamma(x) y\| \leqq(1+\alpha)^{-1} K B^{1+\alpha}\|y\|^{1+\alpha}+C B\|y\| \leqq q\|y\|
\end{gathered}
$$

for $y \in S_{r}$, where $q$ is arbitrary with $B C<q<1$ and

$$
(1+\alpha)^{-1} K B^{1+\alpha}\|y\|^{\alpha}+B C \leqq q
$$

if $\|y\| \leqq r$, i.e., $r^{\alpha}=(q-B C)(1+\alpha) / K B^{1+\alpha}$. Hence, by Lemma $3.1, \Gamma$ is a bounded directional contractor for $P$.

THEOREM 3.1. Under the hypotheses of Lemma 3.2, $P$ is a mapping onto $Y$.

Proof. The proof follows immediately from Theorem [3] and Lemma 3.2.

LEMMA 3.3. Let $P: X \rightarrow Y$ be a nonlinear operator and let $T: X \rightarrow Y$ be differentiable in the Frèchet sense at each $x \in X$. Moreover, suppose that

$$
\left\|T^{\prime}(x)-T^{\prime}(\bar{x})\right\| \leqq K\|x-\bar{x}\|^{\alpha}, \quad 0<\alpha \leqq 1,
$$

$\left\|T^{\prime}(x)^{-1}\right\| \leqq B$ and

$$
\|(P x-T x)-(P \bar{x}-T \bar{x})\| \leqq C\|x-\bar{x}\|
$$

for all $x, \bar{x} \in X$, where $C$ is a constant such that $B C<1$.

Then $\Gamma$ defined by $\Gamma(x)=T^{\prime}(x)^{-1}$ is a bounded directional contractor for $P$.

Proof. We have

$$
\begin{aligned}
\| P(x+ & \Gamma(x) y)-P x-y\|\leqq\| T(x+\Gamma(x) y)-T x-T^{\prime}(x) \Gamma(x) y \| \\
& +\|[P(x+\Gamma(x) y)-T(x+\Gamma(x) y)]-[P x-T x]\| \\
\leqq & (1+\alpha)^{-1} K\|\Gamma(x) y\|^{1+\alpha} \\
& +C\|\Gamma(x) y\| \leqq(1+\alpha)^{-1} K B^{1+\alpha}\|y\|^{1+\alpha}+C B\|y\|
\end{aligned}
$$




$$
\begin{aligned}
& \leqq\left[(1+\alpha)^{-1} K B^{1+\alpha}\|y\|^{\alpha}+C B\right]\|y\| \\
& \leqq\left[(1+\alpha)^{-1} K B^{1+\alpha} r+C B\right]\|y\| \leqq q\|y\|,
\end{aligned}
$$

if $\|y\| \leqq r$, where $q$ is arbitrary with $B C<q<1$ and $r=$ $(q-B C)(1+\alpha) / K B^{1+\alpha}$. Hence, by Lemma $3.1, \Gamma$ is a bounded directional contractor for $P$.*

THEOREM 3.2. Under the hypotheses of Lemma 3.3, $P$ is a mapping onto $Y$.

Proof. The proof follows immediately from Theorem [3] and Lemma 3.3.

LEMmA 3.4. Let $P: X \rightarrow Y$ be a nonlinear operator differentiable in the Fréchet sense with Hölder continuous derivative $P^{\prime}(x)$ satisfying (3.3). Let $\Gamma(x): Y \rightarrow X$ be a bounded linear operator and let $C$ be a positive constant such that

$$
\left\|P^{\prime}(x) \Gamma(x)-I\right\| \leqq C<1
$$

for all $x \in X$, where $I$ is the identity mapping of $Y$. If, in addition,

$$
\|\Gamma(x)\| \leqq B
$$

for all $x \in X$, then $\Gamma$ is a bounded directional contractor for $P$.

Proof. We have

$$
\begin{aligned}
\| P(x+ & \Gamma(x) y)-P x-y\|\leqq\| P(x+\Gamma(x) y)-P x-P^{\prime}(x) \Gamma(x) y \| \\
& +\left\|P^{\prime}(x) \Gamma(x) y-y\right\| \\
\leqq & (1+\alpha)^{-1} K\|\Gamma(x) y\|^{1+\alpha}+C\|y\| \leqq(1+\alpha)^{-1} K B^{1+\alpha}\|y\|^{1+\alpha}+C\|y\| \\
\leqq & {\left[(1+\alpha)^{-1} K B^{1+\alpha}\|y\|^{\alpha}+C\right]\|y\| \leqq\left[(1+\alpha)^{-1} K B^{1+\alpha} r^{\alpha}+C\right]\|y\| } \\
\leqq & q\|y\|,
\end{aligned}
$$

if $\|y\| \leqq r$, where $q$ is arbitrary with $C<q<1$ and $r=$ $(q-C)(1+\alpha) / K B^{1+\alpha}$. Hence, by Lemma 3.1, $\Gamma$ is a bounded directional contractor for $P$.

THEOREM 3.3. Under the hypotheses of Lemma 3.4, P is a mapping onto $Y$.

Proof. The proof follows immediately from Theorem [3] and Lemma 3.4. 
4. Directional contractions. A generalization of the notion of the contraction mapping in a Banach space can be given which is based on the concept of contractor direction.

Definition 4.1. Let $X$ be a Banach space. A mapping $F: X \rightarrow X$ is called a directional contraction if there exists a positive number $q<1$ which has the following property.

For arbitrary $x, y \in X$, there exists a positive number $\epsilon=\epsilon(x, y) \leqq 1$ such that

$$
\|F(x+\epsilon y)-F x\| \leqq q \epsilon\|y\|
$$

THEOREM 4.1. Suppose that $F: X \rightarrow X$ is a directional contraction and $P$ is a closed operator, where $P x=x-F x$. Then $P$ maps the Banach space $X$ onto itself.

Proof. The proof follows from Theorem 2.2. In fact, if $y \in Y=X$ is arbitrary and $\epsilon$ is such that satisfies condition (4.1), then (2.1) is satisfied with $\bar{x}=x+\epsilon y$. Hence, if follows that $\Gamma_{x}(P)=X$. The additional requirement that $\|\bar{x}-x\| \leqq B \epsilon\|y\|$ is also satisfied with $B=1$.

Remark 4.1. It is easy to show that Theorem 4.1 follows immediately from the basic directional contractor theorem (Theorem [4]). In fact, condition (4.1) coincides with the contractor inequality (3.1), where $P x=x-F x$ and $\Gamma(x)=I$ for all $x \in X$. This implies that the identity operator $I$ is a bounded contractor for $P=I-F$.

The following fixed point theorem can be proved as a particular case of Theorem 2.3.

THEOREM 4.2. Let $S$ be an open sphere with radius $r$ and center $\theta$ (zero) in Banach space $X$ and let $F: \bar{S} \rightarrow X$ be a directional contraction on $S$, i.e., for each $x \in S$ and $y \in X$ with $\|y\| \leqq r$, there exists a positive $\epsilon=\epsilon(x, y) \leqq 1$ satisfying (4.1) and $x+\epsilon y \in S$. If $P$ is closed, where $P x=x-F x$ and $r>\|F \theta\| /(1-q)$, then there exists an element $x^{*} \in \bar{S}$ such that $x^{*}=F x^{*}$.

Proof. It is easy to see that the hypotheses of Theorem 2.3 are satisfied with $x_{0}=\theta$. Oviously, $\Gamma_{x}(P)=X$ and condition (3) of Theorem 2.3 that $r \geqq B(1-\bar{q})^{-1}\left\|P x_{0}\right\|$ is satisfied with $B=1$ and $\bar{q}$ being chosen so as to satisfy

$$
q<\bar{q}<1 \quad \text { and } \quad r>\|F \theta\| /(1-\bar{q})
$$


5. Evolution equations. The directional contraction method can be applied to prove an existence theorem for nonlinear evolution equations.

Consider the initial value problem

$$
\frac{d x}{d t}=F(t, x), \quad 0 \leqq t \leqq T, \quad x(0)=\xi
$$

where $x=x(t)$ is a function defined on the real interval $[0, T]$ with values in the Banach space $X$, and $F:[0, T] \times X \rightarrow X$. Denote by $X_{T}$ the space of all continuous functions $x=x(t)$ defined on $[0, T]$ with values in $X$ and with the norm $\|x\|_{C}=\max [\|x(t)\|: 0 \leqq t \leqq T]$.

Instead of (5.1), we consider the integral equation

$$
x(t)-\int_{0}^{t} F(s, x(s)) d s=\xi
$$

as an operator equation in $X_{T}$ and we assume that the integral operator is closed in $X_{\mathrm{T}}$.

Suppose that there exists an integrable function $K(t), 0 \leqq t \leqq T$, which has the following property. For arbitrary $x, y \in X_{T}$ there exists a positive number $\epsilon=\epsilon(x, y) \leqq 1$ such that, for all $0 \leqq s \leqq T$, we have

$$
\|F(s, x(s)+\epsilon y(s))-F(s, x(s))\| \leqq K(s) \epsilon\|y\|_{c} .
$$

THEOREM 5.1. Suppose that the integral operator in (5.2) is closed in $X_{T}$ and that there exists an integrable function $K(t)$ which satisfies (5.3) and such that

$$
\int_{0}^{T} K(t) d t=q<1 .
$$

Then for arbitrary $\xi \in X$ equation (5.2) has a continuous solution $x(t)$.

Proof. It is easily seen that condition (5.3) implies condition (4.1) so that the integral operator in (5.2) yields a directional contraction in $X_{T}$. Thus, the proof follows immediately from Theorem 4.1.

Definition 5.1. Let $F(t, x)$ be continuous in $(t, x) \in[0, T] \times X$, and suppose that there exists an integrable function $K(t), 0 \leqq t \leqq T$, which has the following property.

For each $t \in[0, T]$ and $x, y \in X$ there exists a positive $\epsilon=\epsilon(x, y) \leqq$ 1 such that 


$$
\|F(t, x+\tau y)-F(t, x)\|<K(t) \tau\|y\|
$$

for all $0 \leqq \tau \leqq \epsilon=\epsilon(x, y) \leqq 1$.

Then $F$ is said to be Lipschitz demi continuous.

Let us suppose that $F$ satisfies the following hypothesis.

Given two arbitrary continuous functions $x=x(t)$ and $y=$ $y(t) \in X_{T}$, there exists a sequence $\left\{\epsilon_{n}\right\}$ convergent to zero such that for any $\eta>0$, there is a positive $\delta$ which satisfies the following condition for almost all $n$.

$$
\epsilon_{n}^{-1} \int_{\omega}\left\|F\left(s, x(s)+\epsilon_{n} y(s)\right)-F(s, x(s))\right\| d s<\eta,
$$

where $\omega \subset[0, T]$ is an arbitrary subset of $[0, T]$ with measure $\mu(\omega)<\delta$.

THeorem 5.2. Suppose that $F:[0, T] \times X \rightarrow X$ is Lipschitz demi continuous and satisfies conditions (5.4) and (5.6). Then the initial value problem (5.1) has a solution.

Proof. Let us consider the equivalent problem of solving the integral equation (5.2) for arbitrary $\xi \in X$. The proof will follow from Theorem 4.1 if we can show that the integral operator is a directional contraction. In other words, we have to show that $\epsilon=\epsilon(x, y) \leqq 1$ in (5.3) depends on the continuous functions $x, y \in X_{T}$. Let $\bar{q}$ be arbitrary with $q<\bar{q}<1$, where $q$ is defined by (5.4). Given arbitrary continuous functions $x=x(t), y=y(t) \in X_{T}$, put in (5.6) $\eta=(\bar{q}-q)\|y\|_{C}$ and let $\delta$ correspond to $\eta$. By a theorem of Lusin there exists an open subset $\omega \subset[0, T]$ with measure $\mu(\omega)<\delta$ such that the function $K$ is continuous on $[0, T] \backslash \omega$. It follows from (5.5) that for each interior point $s \in$ $[0, T] \backslash \omega$, there exists an open subset $\Delta(s) \subset[0, T] \backslash \omega$ such that

$$
\|F(t, x(t)+\tau y(t))-F(t, x(t))\|<K(t)\|y\|_{C}
$$

for all $t \in \Delta(s)$ and $0 \leqq \tau \leqq \epsilon=\epsilon(x(s), y(s)) \leqq 1$. Let $\left\{\Delta\left(s_{i}\right)\right\}, \quad i=$ $1,2, \cdots, n$, be a finite subcovering of $[0, T] \mid \omega$, and put

$$
\epsilon_{0}=\epsilon(x, y)=\min \left[\epsilon\left(x\left(s_{\imath}\right), y\left(s_{\imath}\right)\right): 1 \leqq i \leqq n\right],
$$

where $x, y \in X_{r}$. Then we have

$$
\|F(t, x(t)+\tau y(t))-F(t, x(t))\|<K(t)\|y\|_{C}
$$

for all $t \in[0, T] \backslash \omega$ and $0 \leqq \tau \leqq \epsilon_{0} \leqq 1$. Now, denote by $\epsilon$ the number $\epsilon_{k} \leqq \epsilon_{0}$. Such $\epsilon_{k}$ exists, since $\epsilon_{n} \rightarrow 0$ as $n \rightarrow \infty$. Then it follows from (5.6) and (5.7) that 


$$
\begin{aligned}
& \int_{0}^{t}\|F(s, x(s)+\epsilon y(s))-F(s, x(s))\| d s \\
& \leqq \int_{0}^{T}\|F(s, x(s)+\epsilon y(s))-F(s, x(s))\| d s \\
&= \int_{[0, T] \omega}\|F(s, x(s)+\epsilon y(s))-F(s, x(x))\| d s \\
& \quad+\int_{\omega}\|F(s, x(s)+\epsilon y(s))-F(s, x(s))\| d s \\
& \leqq \epsilon \int_{0}^{T} K(s) d s\|y\|_{C}+(\bar{q}-q) \epsilon\|y\|_{C} \\
&=\bar{q} \epsilon \|
\end{aligned}
$$

Hence, it follows that the integral operator in (5.2) is a directional contraction.

REMARK 5.1. If the function $K(t)$ is continuous on $[0, T]$, then condition (5.6) is superfluous.

Acknowledgment. Professor W. A. Kirk has kindly informed me that he and J. Caristi have proved a result which is similar to Theorem 1.1 and is to be published in the Bull. de l'Acad. Polonaise des Sci.

\section{REFERENCES}

1. M. Altman, A generalization of Newton's method, Bull. Acad. Polon. Sci., Cl. III, 3 (1955), 189-193.

2. Inverse differentiability, contractors and equations in Banach spaces, Studia Math., 46 (1973), 1-15.

3. - Directional contractors and equations in Banach spaces, ibid., 101-110.

4. Solvability of nonlinear operator equations, to appear.

5. F. E. Browder, Normal solvability and the Fredholm alternative for mappings into infinite dimensional manifolds, J. Functional Analysis, 8 (1971), 250-274.

6. - On the Fredholm alternative for nonlinear operators, Bull. Amer. Math. Soc., 76 (1970), 993-998.

7. J. Kolomý, Normal solvability, solvability and fixed-point theorems, Colloquium Math., 29 (1974), 253-266.

8. S. I. Pokhozhaev, Funktsion, Analiz i Ego Prilozhen, 3 No. 2 (1969), 80-84 (see translation from Russian).

9. P. P. Zabreiko and M. A. Krasnosel'skii, Solvability of nonlinear operator equations, ibid., 5, No. 3 (1971), 42-44.

Received June 10, 1975 and in revised form October 7, 1975. 



\section{Pacific Journal of Mathematics}

\section{Vol. 62, No. $1 \quad$ January, 1976}

Mieczyslaw Altman, Contractor directions, directional contractors and

directional contractions for solving equations . .................. 1

Michael Peter Anderson, Subgroups of finite index in profinite groups .........

Zvi Arad, Abelian and nilpotent subgroups of maximal order of groups of odd order

John David Baildon and Ruth Silverman, On starshaped sets and Helly-type theorems ..........................................

John W. Baker and R. C. Lacher, Some mappings which do not admit an

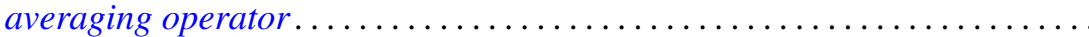

Joseph Barback, Composite numbers and prime regressive isols . . . . . . . . . .

David M. Boyd, Composition operators on $H^{p}(A) \ldots \ldots \ldots \ldots \ldots \ldots \ldots$

Maurice Chacron, Co-radical extension of PI rings . . . . . . . . . . . . .

Fred D. Crary, Some new engulfing theorems . . . . . . . . . . . . . . .

Victor Dannon and Dany Leviatan, A representation theorem for convolution transform with determining function in $L^{p} \ldots \ldots \ldots \ldots \ldots \ldots \ldots \ldots \ldots \ldots \ldots \ldots \ldots \ldots$

Mahlon M. Day, Lumpy subsets in left-amenable locally compact

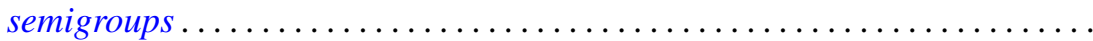

Michael A. Gauger, Some remarks on the center of the universal enveloping algebra of a classical simple Lie algebra . .

David K. Haley, Equational compactness and compact topologies in rings

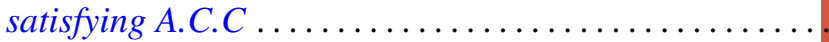

Raymond Heitmann, Generating ideals in Prüfer domains .

Gerald Norman Hile, Entire solutions of linear elliptic equations with

Laplacian principal part. .

Richard Oscar Hill, Moore-Postnikov towers for fibrations in which $\pi_{1}$ (fiber) is non-abelian

John Rast Hubbard, Approximation of compact homogeneous maps . .

Russell L. Merris, Relations among generalized matrix functions . .

V. S. Ramamurthi and Edgar Andrews Rutter, On cotorsion radicals ...

Ralph Tyrrell Rockafellar and Roger Jean-Baptiste Robert Wets, Stochastic

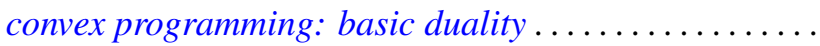

Alban J. Roques, Local evolution systems in general Banach spaces ..

I. Bert Russak, An indirect sufficiency proof for problems with bounded state variables.

Richard Alexander Sanerib, Jr., Ultrafilters and the basis property. .

H. A. Seid, The decomposition of multiplication operators on $L_{p}$-spaces . .

Franklin D. Tall, The density topology .................. 